\title{
An Efficient Scheme for Vein Detection using Accuvein Apparatus Based on Near Infrared with Broadcom Chip
}

\author{
Sahana D S, Dayanand Lal. N, Vidya J, Bhanujyothi H C
}

\begin{abstract}
Venipuncture has been considered as one of the main fitness analysis strategies. Even although venipuncture has been taken into consideration as one of the highest prioritized and commonly accompanied practised in hospitals is to carry out obtain the veinsfor small kids, elderly peoples, fat , anaemic, or darkish skin colored patients has been a difficult procedure for medical practitioners. To get the solution for this problem, many devices using infrared light have became today\&\#39;s trend. But those devices share a commonplace drawback, for visualizing deep veins or veins of a thicker part of subject like limb. This paper clarifies a vein-picturing device, Accuvein which uses Near infrared (NIR) light. The light inventory quickly extends straightforwardly to the chose selected part of the skin. The camera sensor has been used to come across infrared radiation to take the vein photos. With the addition of an image processing process, the first-class of vein shape obtained is more desirable showing it extra as it should be. The implemented device has met the requirements of a desired output image when limb areas kept under Accuvein device obtaining the efficient images. The visibility of veins for the purpose of Cannulation increased by using Accuvein device.
\end{abstract}

Keywords : Cannulation, CCD Camera, Near-infrared light, Phlebotomists, Venipuncture.

\section{INTRODUCTION}

A major means for treating for any diseases is to go with blood taken from veins. This involves an incursive method of Cannulation that needs proper selection of vein[1]. Phlebotomist, the one who makes an incision in a vein with a needle, uses a vein finder that helps to effortlessly discover the vein by maintaining a strategic distance from pre-expository mistake in the example assortment and to reduce the uneasiness and pain to the patient. Venipuncture or Venepuncture is a process followed for getting blood from

Revised Manuscript Received on February 05, 2020.

* Correspondence Author

Sahana D S, Department of CSE, GITAM School of Technology,Bengalure, India. Email: ds.sahana16@gmail.com

Dayanand Lal. N, Department of CSE, GITAM School of Technology,Bengalure, India... Email: dayanandlal@gmail.com

Vidya J, Department of CSE, GITAM School of Technology, Bengalure, India. Email: vidya.sjprakash@gmail.com

Bhanujyothi H C, Department of CSE, GITAM School of Technology, Bengalure, India. Email: banu.banuchandrashekar@gmail.com

(C) The Authors. Published by Blue Eyes Intelligence Engineering and Sciences Publication (BEIESP). This is an open access article under the CC BY-NC-ND license (http://creativecommons.org/licenses/by-nc-nd/4.0/) medical laboratory testing, which primitively uses sample of

vein, which needs to be done regularly during medical checkups. However, the person who is fat or dark skin colour will experience 'hypodermic' while they are performing the visualization of vein structures. For those people the phlebotomist[2] will not be having any other choice sporting out a visually impaired stick dependent on anatomical information with patients revel in. This may also lead to direct or indirect damage to the patient who undergoes venepunture, which may additionally once in a while cause loss of life of a affected person . Even it is miles simpler to decide out the vein structures at the hand-ventral or arm, genuinely we will try to keep away from the aspect results which may additionally cause very extreme risk elements of phlebotomy errors, however additionally try to lessen an attempt required in phlebotomy.

The usage of Venepuncture in our everyday health care procedure has been increased drastically. The literature and look for on difficulties of Venepuncture and blood assortment bring about numerous unpredictable issues Problems that might get up from venepuncture encompass ; haematoma formation, nerve harm, ache, haema-attention, more vasation, iatrogenic anaemia, arterial puncture, petechiae, allergies, fear and phobia, contamination, syncope and fainting, excessive bleeding, edema and thrombus[3]. There may be numerous difficulties confronted by means of phlebotomists, medical attendants and pathologists while recognizing a patient's vein for venepuncture. Heat Burns and different injuries for a body make it complicated to find veins and positioned the tablets to shop the lifestyles. If in case of blood transfusion or withdrawal, we want to test for the placement of the veins. Sometimes the skilled nurses and doctors might also sense it hard to exactly get the blood veins on the primary strive.

Inappropriate puncturing of veins might lead to many problems like swelling, bleeding or permanently damaging the veins, mainly for aged and for babies[4]. To conquer the troubles various devices had been evolved by using overcoming the demanding situations like cost ,portability factor and efficiency for infrared imaging detection system. The design of the vein detection system have to be an easy-to-enforce device which makes use of a IR sensitive camera that takes a photograph of the objects vein underneath a source of infrared radiation with a precise wavelength which is illustrated in Fig.1.Burns and various wounds for a body make it confused to discover veins and situated the tablets to shop the ways of life. 
In the event that if there should arise an occurrence of blood transfusion or withdrawal,

we need to test for the situation of the veins.

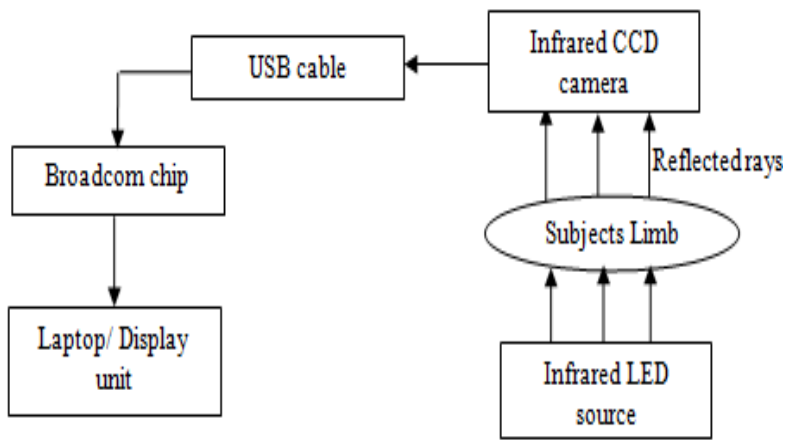

Fig. 1. NIR Vein Imaging System.

In a few scientific situations, the place of vein desires to be recognized. Each second counts while docs are treating trauma sufferers.

Some of the other devices which help the venipuncture procedure to perform the visualizing of the vein patterns exist. The techniques uses near-infrared (NIR) light or far-infrared (FIR) light[6]. NIR is used because of its high benefits like low cost and safety . By effectively utilizing these two kinds of lights in CCD camera techniques, scientists can get the pictures of objects that extent from imagining vein structures until the division. For the FIR light based technique [7], a camera is expected to make the water ingestion. It is also helpful to collect the high quality images for segmentation. The only disadvantage of FIR light based technique is high cost. A replacement can be taken expensive FIR method as the NIR light that ranges from (700 nm-2500 $\mathrm{nm})$ are used to visualize the vein pattern[8]

The NIR approach follows two kinds of image collecting systems of veins. (i) Reflection and (ii) Penetration, as shown in fig 2(a) and fig2(b) . The very first method, reflection technique is mainly affected by reflected lighting fixtures, the photos we get seem to be brighter. So simplest, it is having lesser comparison and visibility. The penetration approach is tons increasingly useful, while identifying vein structures on the appendage as light can't enter to a much profundity in a grown-up's arm. Majority of vein visualizing devices use the above strategies of NIR light. Some of the examples like Vein viewer and Accuvein put an image in green and red photo respectively at the pores and skin by means of the usage of the reflection method.

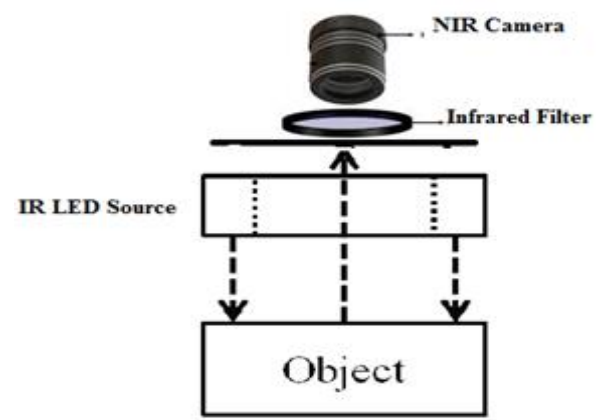

Fig.2 (a). Reflection model of image collecting system.

The devices need darker settings for projection. Similarly, the images generated by penetration method are less accurate which purely depend on the projection angle say for example,
Trans-illuminator Device, Vein Navigation Device and Vascu-Luminator [2].

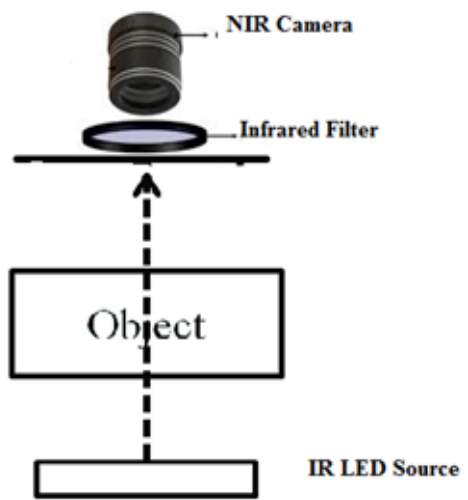

Fig.(b). Penetration model of image capturing system.

The problem with the penetration method is, the pictures obtained will not be much accurate and clear because the arm is not present at a significant depth. Limitation of the Penetration strategy is that, on the off chance that we remember thicker body component like arm, the NIR light can't accomplish the opposite side of the arm. The explanation is that every one trans-illuminated NIR light may be absorbed sooner than entering to the next part of the arm.

\section{RELATED WORK}

L.Wang et al[9] have proposed a method on non-invasive technique to get the image of veins patterns evaluated using FIR imaging technique. The analysis of the data collected using FIR(Far infrared) imaging technique, is less successful as the images of palm/limb/wrist obtained have covered lesser features of veins and also the quality of images obtained are not much accurate as shown in figure 4. FIR will be operative by detecting electromagnetic radiations emitted from human body. And also images that captures the large veins are very less sensitive to ambient environmental factors like temperature, humidity conditions and human body temperature[10].

Soujanya Ganesh[11] has viewed how an ultrasound system that fits for photographs might to consider the statistics like how much deep it can reach, what might be the size, in which place object is placed , the complete structures obtained to get the guidance on locating the veins which are abnormal. System works on the idea of getting the ultrasonic waves by the method of transmission on to the human frame by using the device called transducer to become aware of the reflected rays from the gadgets internal. The transducer discovers the light rays which would converted into electrical energy. Devices operating on Trans-illuminating light assets mechanisms want lights to be switched off for the medical doctors to get the images of veins simple and clear way. Markers are utilized on the patient's skin after observing the vein. Very high resolution ultra vein scanners are needed by the transducer which need to be held while needle insertion

seem to be an uncomfortable way to get accurate view on ultrasound display. 
Naoya Tobisawa et al[12] describes the framework which involves the exceptionally high-power and low-level light source, the method functions as follows: the near infrared CMOS camera and little one-eye head put on the showcase to display. Utilizing this framework, they get the trans-illumination pictures of the subject's limb. They can acquire a more clear trans-illuminated images of profound situated veins, which is utilized to help the IV infusions, the varicose-vein treatment, blood vessel catheterization, intra are employable perception of veins and lymph conduits, etc. Since it utilizes low level light sources, here and there it is preposterous to expect to get a reasonable and a decent quality pictures.

Phlebotomycoach[13] is a technique which illustrates Venepunture ,as taking a sample of blood from a vein from hand. If patient requires frequent blood tests or medication continuously, the technique of intravenous (IV) comes in to noticing the presence of veins, so that the medications or fluids are given intravenously. This requires a very good skilled medical technician called phlebotomist, to follow up the procedure painlessly. But some of the patients are very feared about this procedures due to which they might feel light headache, some patients might undergo fainting, if the phlebotomist is not much experienced he might have pricked the wrong nerve which may lead to damaging the nerve, a severe blood cloths, infections and many more.

F. B. Chiao et al[14] explains the characteristics of the patients that identifies veins available for the purpose of IV access. The qualities considered depend on fat patients crew who are related with lesser vein visibility. Test directed on fat patients, gave the after effects of roughly six veins on every furthest point dependent on unassisted vision . Utilization of the new infrared VueTek Veinsitew results inside the perceivability of shallow veins qualified for IV Cannulation which can be raised while utilizing. Prior identity of patients with hard IV get admission to could deliver the higher clinical care, with the aid of stopping complications lowering the ache for patients.

Cheng-Tang Pan et al [15],illustrates (i) commonly used method for diagnosis through medical laboratory testing that makes use of blood as a sample (ii) Uses the concept of invasive method called Cannulation which gives the viability to properly select the veins. Usage of devices like vein finder helps the physicians to locate the veins easily, which prevent the problems that would occur in collecting the samples reducing the discomfort and pain of the patient. Due to the experimental results, based on the consideration of various human factors, besides taken huge number of aspects and patients for testing the actual devices and model yielded the better results.

\section{METHODOLOGY}

\section{A. About VEIN DETECTION}

In case of emergency when the patients are admitted in to hospital each and every second counts. Physical injuries might make the physicians and phlebotomist feel difficult to find veins on the first try, in particularly for obese people to supply the drugs. Here comes the actual picture of detecting the veins and to know how difficult it is to get the clear picture. In this procedure, the skin on hand will be pricked by

picture of veins. If we take the example of having pattern of blood for diagnosing, blood transfusion, blood donation, and so on. It's far crucial to know the exact region of the veins[14]. In numerous scientific emergencies, the precise location of veins desires to be diagnosed like Intravenous injections(IV), Bruises and Bums, Blood transfusions, Blood donation, kidney dialysis and also locating veins in small kids and infants seems to be very difficult to prick them several times with a needle is one of the crucial thing for infants, Geriatrics: for aged people are required repetitive blood tests or medicinal injections.

\section{B. Idea Of Near-Infrared(NIR) Imaging}

The portion of the spectrum which is visible to the human eye is called visible spectrum. Electromagnetic radiation is called as visible light. Persons eyes is able to see with the wavelengths from about 380 to $740 \mathrm{~nm}$. To view the images of vein patterns around the boundary, the chance of viewing below everyday visible light conditions may be very less. For this scenario, the solution can be using Near-Infrared imaging(NIR) techniques. The features of NIR which is used to detect the veins are as follows:

1. NIR can do the penetration into the subject's tissue till $3 \mathrm{~mm}$ beneath [15].

2. If the content of haemoglobin has reduced in the subjects body, it absorbs more infrared radiation rather than its surrounding tissue. So setting infrared radiation at the preferred a part of the frame we can acquire the vein photo captured through an IR camera. In the resulting picture, the veins appear to be darker than the surrounding tissue. Near to Infrared (NIR) radiation of the wavelength district 740 $\mathrm{nm}-760 \mathrm{~nm}$ can distinguish on veins anyway not courses because of the particular ingestion of infrared radiation in blood vessels[16].The illustration has also been studied under drug reactions that need to evaluated by considering the reactions on the subjects body when it has been injected in to veins[17].

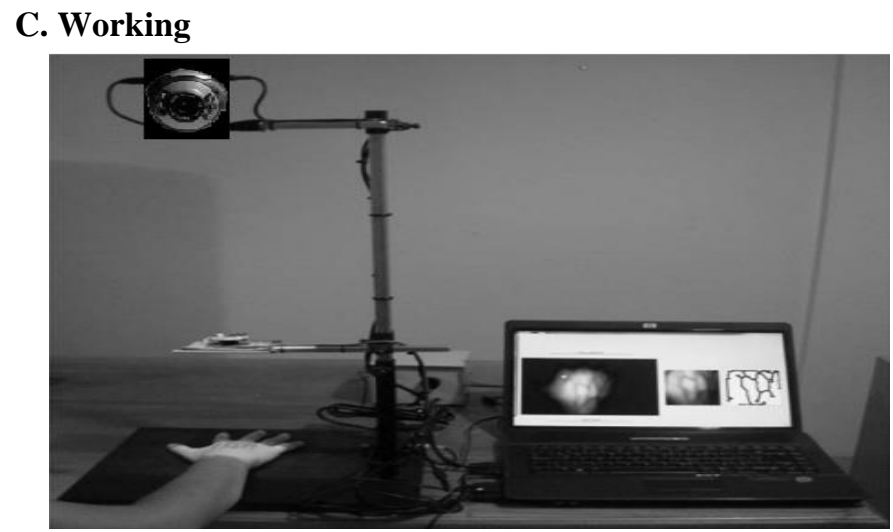

Fig.3. Prototype of experiment.

i. As shown in fig.3, IR light will be projected on the subject's skin/limb, which penetrates into tissues and veins and its surroundings tissues and a part of this light will be reflected. The reflected light rays have been captured by a IR camera. In NIR, the ring of LEDs are used to get the clear image of the preferred frame component with infrared light.

This ring constitutes a circuit driver and the power supply could be provided from the pc the usage of a USB cable[17]. Below fig.4 shows the IR 
light Source Setup. The main objective of using IR light source is to get the good vein images, facilitating an accurate comparison between veins and its surrounding tissue.
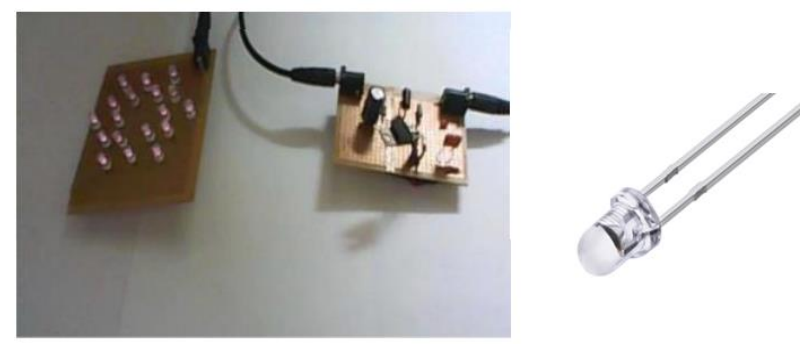

Fig.4. IR light source.

ii. This reflected light rays have been captured by an IR camera. CCD cameras are chosen for NIR because of its sensitivity to IR, which gives a better picture quality. One more advantage is camera being cost effective i.e lesser cost. The camera really works well on laptop which uses windows OS. The CCD camera has sufficient spatial resolution to get clear vein pictures. CCD cameras as shown in fig.5, have $1000 \mathrm{~nm}$ range of wavelength and the human eye cannot detect wavelengths which is greater than $750 \mathrm{~nm}$.

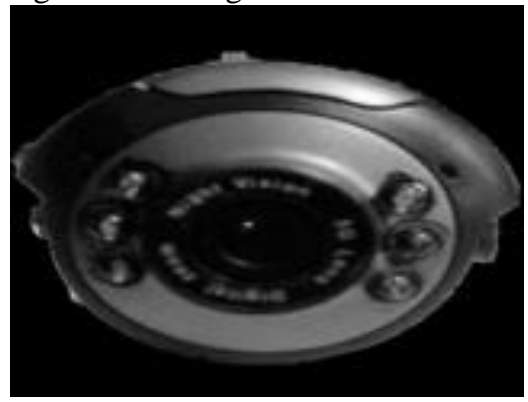

Fig.5. NIR CCD camera.

iii. In Region of Interest(ROI), Raw image captured from the CCD camera will be fed into the ROI Component. ROI is mainly used to select the region of interest that wishes to get . NIR light source when projected shows the complete images which has captured using CCD camera , so only ROI is used to get highlighted segment of an image. Reason to carry out ROI step as utilizing the whole image takes much time and the final captured image might not be much accurate. So ROI is used to acquire the required part of the image.

iv. In Pre-processing, Output of ROI , which have been captured from IR camera will be fed to the Broad-cam chip (Raspberry Pi3), which will carry out the pre-processing steps to get the good quality of images at the end. Pre-processing needs to be performed before passing images to external source as shown in fig.6.

a. Required part of image obtained in ROI step undergoes pre-processing

b. Image processing algorithm has been applied to show the exact vein pattern of arm/wrist/ limb, to remove noise and also to enhance the quality of input image that have acquired. Vascular Imaging Algorithm has shown the process for getting various patterns of blood vessel to get a images which are contrast using the near-infrared imaging system.

c. Segmentation using Threshold : Once ROI outputs the required image part and feeds it to the Broadcom chip, the Image processing algorithm which are embedded in the chip will eliminate noise and there by gives the enhanced images.
Then segmentation will be used to differentiate the vein pattern from the background images. Obtained vein patterns will be considered and isolated from rest of the images. If this step not executed properly, noises will be increased. If we don't get proper images of vein, obviously it will be of no use again. The concept of Thresholding is used to get converted the gray scale vein images to the binary images.

d. Filter: The output of segmentation component consists of gray scale images that might have some unclear and also unwanted shadows in the images of veins which are displayed. So it has fed into the filter to upgrade the nature of image obtained. In order to block the unwanted visible radiations which are below $720 \mathrm{~nm}$ allows the near infrared radiation to fed to the transmission filter . The filter can now effectively block more than $90 \%$ of the visible radiations. The output of this filter will be the enhanced set of images which is of good quality.

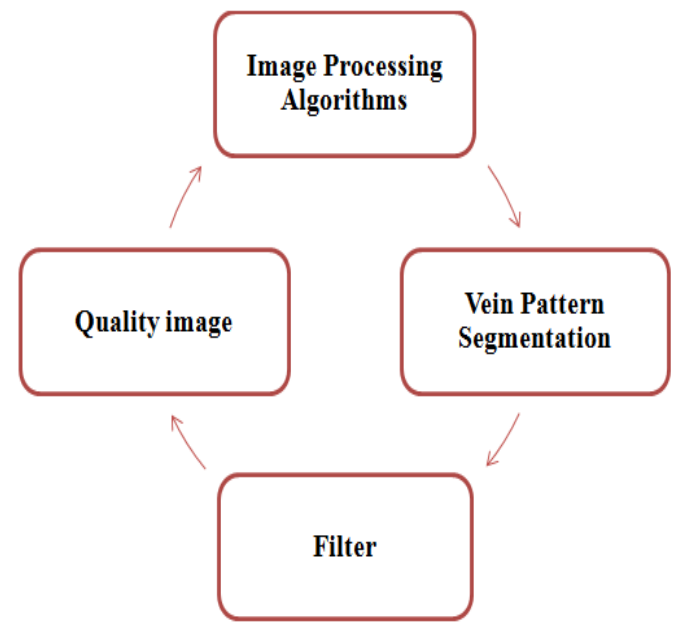

Fig.6. Pre-processing.

v. In Post-processor, the operation of this component is performed after the data received from the external source but before feeding for the display unit. It checks with the database to know whether the obtained image after the processing is matching with the template that is stored in database priory.

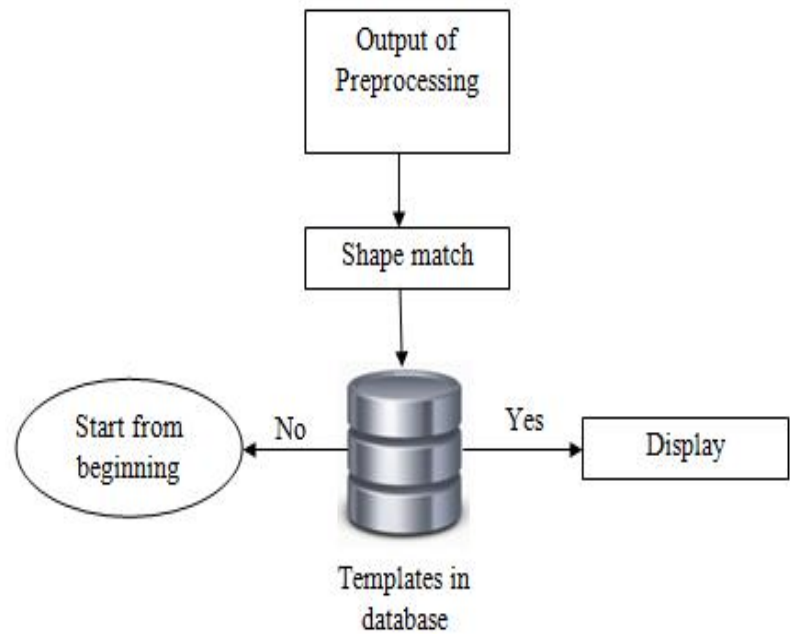

Fig.7. Post processing.

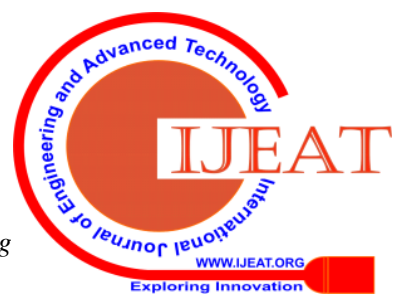


vi. In Display Unit, the main objective of this paper will be considered that is to display the proper vein patterns .The display unit is used to display the final processed image of veins on laptop, which helps to get the clear difference between veins and its back-ground.

\section{Implementation}

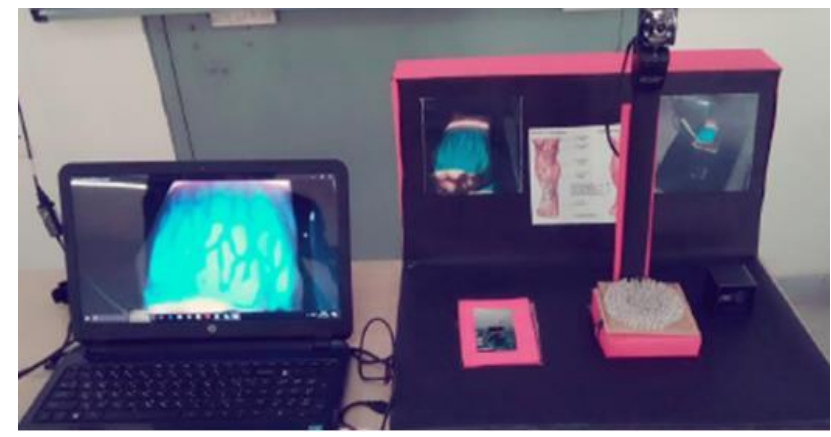

Fig 8: Working model of NIR Based Accuvein

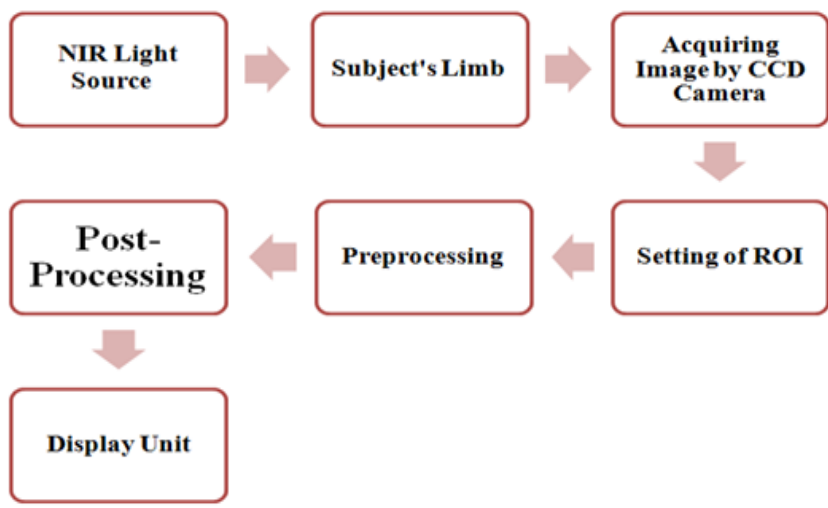

Fig 9: Flow chart of the model

The information captured from CCD camera will be given to the processing unit at different stages to enhance the image visualization using valid image processing algorithms. When it uses the algorithms to enhance the image quality the output obtained with somewhat dark veins, allows for easier differentiation between the veins and its surroundings. After this , a threshold (segmentation) is applied to create a images of color black and white where in it contains the vein patterns. Now the gray scale image will be ready to be segmented. Using the concept of thresholding it is easier to get the boundaries between "foreground" and "background" pixels. Fig 9 shows complete overview of the proposed system model. The processor usually consists of Broad-cam chip and the laptop that contains required software to get effective image patterns. Accuvein device, which is based on NIR , makes use of OPEN CV (Computer Vision) Library which is supported by the Python Programming Language to perform the necessary enhancement of the information obtained from the acquisition system. The Broadcom chip consists of another USB cable interface from the laptop and an Ethernet cable to get connected as an interface between the Broadcom chip and the laptop. The images obtained are then undergo processing using the built in language of the Broadcom chip, which uses Python and is supported by Open CV Image processing library saved on the SD card and that will be inserted into the broadcom chip along with the boot software called Raspbian Jessie. The results of this may provide a binary image which demonstrates the foreground image and the history image based at the contrast greater picture[10].

Filter takes care of removing the shadow and faint images and gives the enhanced vein pattern after this processing step. Then post-processing will be applied to check out the shape match in database, if it is matching it will be displayed in the display unit with much good presentation of vein patterns.

\section{E. Experimental Results}

Fig 10 , fig 11 and fig 12 shows the experimental results. Below figures shows the results obtained when NIR Light falls on limb and re-projected images of palm, wrist and hand.

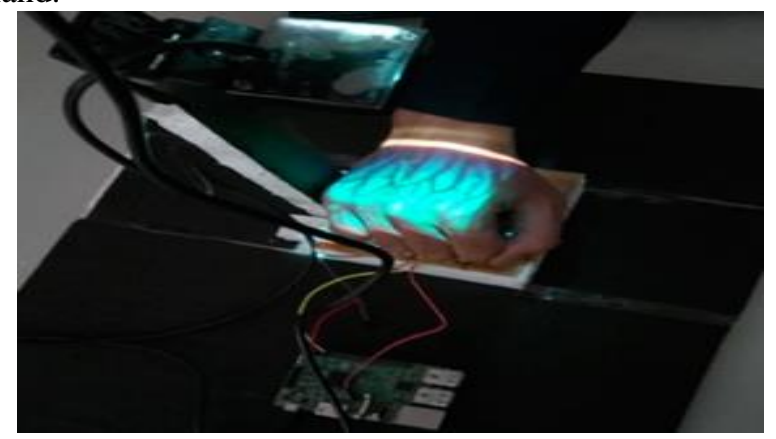

Fig 10 : Image created by the NIR LED array

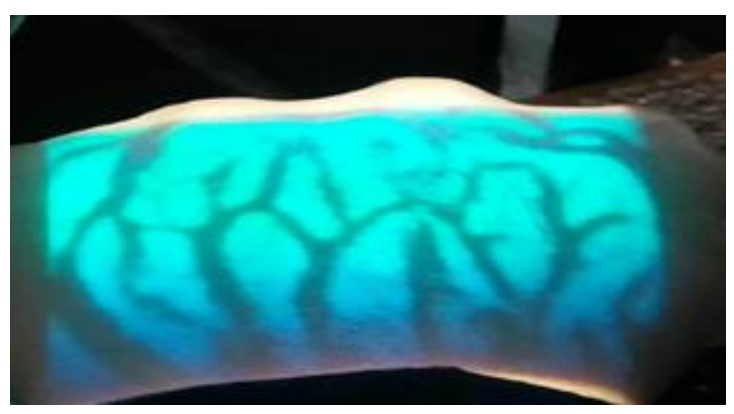

Fig 11 : Re-projection of the captured image on the subject's skin

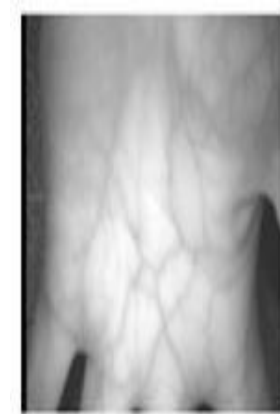

(a) NIR imase of palm

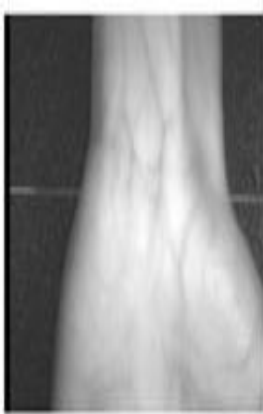

(b) NTR imace of the wist

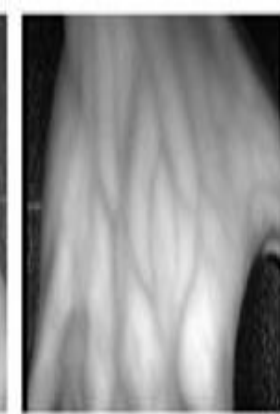

(c) NRR imare of back of the hand
Fig 12 : NIR based Hand images.

\section{COMPARISON CHART WITH TYPE OF SOURCE AND TECHNIQUE USED}


Table 1: Characteristics Chart Comparison

\begin{tabular}{|c|c|c|}
\hline Characteristi:s & Reflection Method & Penetration Method \\
\hline Behaviour & $\begin{array}{c}\text { Can penetrate to } \\
\text { thicker part like am }\end{array}$ & $\begin{array}{c}\text { Cannot penetrate to thicker } \\
\text { part like arm }\end{array}$ \\
\hline Device & $\begin{array}{c}\text { Vein viewer/ } \\
\text { Accuvein }\end{array}$ & $\begin{array}{c}\text { Trans-illuminator device/ } \\
\text { Vein Navigation device }\end{array}$ \\
\hline $\begin{array}{c}\text { Appearance of } \\
\text { Image }\end{array}$ & $\begin{array}{c}\text { Bright image, low } \\
\text { contrast }\end{array}$ & Lesser bright image \\
\hline Source & NIR & LED \\
\hline Image class & $\begin{array}{c}\text { Good quality vein } \\
\text { pattems }\end{array}$ & $\begin{array}{c}\text { Less quality vein pattems } \\
\text { compared to NIR }\end{array}$ \\
\hline
\end{tabular}

\section{CONCLUSION}

This paper explains the overview of near infrared techniques for Vein detection. When IR source projected on subject's skin/limb/wrist, it passes underneath the tissue and veins absorbs that light and the vein will seem darker than surrounding tissue. A good configured CCD camera has been used to get the images of vein. Using the proposed prototype , the trans-illumination images of the subject's skin/limb/wrist will be obtained. By using image processing algorithm , the focus is on getting the enhanced vein image patterns which undergo several pre-processing and post-processing steps by removing all the noises and unnecessary part of images which are present when the image has been captured and achieved. In this manner, the reason to get a productive, non-intrusive device for distinguishing vein images by using near infrared source has been carried out.

\section{REFERENCES}

1. Venepuncture and intravenous cannulation". Clinical Skills, University Section of Anesthesia, Pain and Critical Care Medicine.,www.gla.ac.uk

2. Cheng-Tang Pan,Mark D. Francisco, Chung-Kun Yen 1, Shao-Yu Wang 1 and Yow-Ling Shiue, "Vein Pattern Locating Technology for Cannulation: A Review of the Low-Cost Vein Finder Prototypes Utilizing near Infrared (NIR) Light to Improve Peripheral Subcutaneous Vein Selection for Phlebotomy".

3. Omiepirisa Yvonne Buowari, "Complications of venepuncture".

4. Pranoti Khollam, Dr. Pranoti Mane,"Comparative Analysis of Vein Detection Techniques".

5. Vishal V. Gaikwad, Sanjay A. Pardeshi, " Vein detection using infrared imaging system".

6. Donghoon Kim, Yujin Kim, Siyeop Yoon and Deukhee Lee, "Preliminary Study for Designing a Novel Vein-Visualizing Device".

7. Wang, L.; Leedham, G.; Cho, S ,"Infrared Imaging of Hand Vein Patterns for Biometric Purposes". IET Comput. Vis. 2007, 1, 113-122.

8. Cuper, N.J.; Klaessens, J.H.; Jaspers, J.E.; de Roode, R.; Noordmans, H.J.; de Graaff, J.C.; Verdaasdonk, R.M, "The use of near-infrared light for safe and effective visualization of subsurface blood vessels to facilitate blood withdrawal in children".

9. L.Wang, G Leedham and S Y .Cho, "Infrared imaging of Hand vein patterns for biometric purposes".

10. C.-L Lin and K.-C. Fan, "Biometric Verification Using Thermal Images Of Palm-dorsa Vein Patterns",. IEEE Trans. Circuits and Systems for Video Technology, 2004. 14(2): p. 199-213.

11. Soujanya Ganesh, "Depth and Size Limits for the Visibility of Veins Using the VeinViewer Imaging System", University of Tennessee Health Science Center.

12. Naoya Tobisawa, Takeshi Namita, Yuji Kato and Koichi Shimizu ,"Injection Assist System with Surface and Transillumination Images". IEEE 2011.

13.https://phlebotomycoach.com/faqs/what-is-venipuncture

14.F. B. Chiao, F. Resta-Flarer, J. Lesser, J. Ng, A. Ganz, D. Pino-Luey, H. Bennett, C. Perkins Jr and B. Witek , "Vein visualization: patient characteristic factors and efficacy of a new infrared vein finder technology",

15.Cheng-Tang Pan 1,2,y, Mark D. Francisco 1,3,4,y, Chung-Kun Yen 1, Shao-Yu Wang 1 and Yow-Ling Shiue 3, "Vein Pattern Locating Technology for Cannulation: A Review of the Low-Cost Vein Finder Prototypes Utilizing near Infrared (NIR) Light to Improve Peripheral Subcutaneous Vein Selection for Phlebotomy",

16.Manam Mansoor, Sravani.S.N, Sumbul Zaha Naqvi, Imran Badshah, \& Mohammed Saleem, "Real-time cost infrared vein imaging system" Proceedings of the IEEE International conference on Signa Processing, Image Processing, \& Pattern Recognition (ICSIPR 2013).

17.Sahana D S, Prof Shantala C P , Girish L, "Automatic Drug Reaction Detection Using Sentimental Analysis",

18.Wang Lingyu and Graham Leedham, "Near- and Far- Infrared Imaging for Vein Pattern Biometrics ", Proceedings of the IEEE International Conference on Video and Signal Based Surveillance (AVSS 2006).

19.Liukui Chen, Hong Zheng, Li Li, Peng Xie \& Shuo Liu, "Near-infrared Dorsal Hand Vein Image Segmentation by Local Thresholding Using Grayscale Morphology", 1-4244-1120-3/07, IEEE-2009.

20.Sang-Kyun Im, Hyung-Man Park, Young-Woo Kim, Sang-Chan Han, Soo-Won Kim, Chul-Hee Kang andChang-Kyung Chung, "An Biometric identification system by extracting hand vein patterns", Journal of the Korean Physical Society, 38(3): 268-272, March 2001

21.P Sannidhi, Sathwik R Gutti, MR Shamanth, R Sai Charan, SK Parikshith Nayak, "Image Stitching of Dissimilar Images", International Journal of Research in Engineering, Science and Management vol-2, issue-5, 2019.

\section{AUTHORS PROFILE}

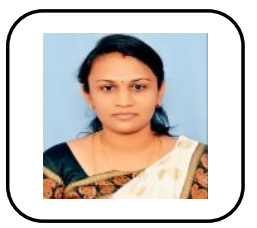

Mrs. Sahana D S is working as an Assistant Professor in the department of Computer Science and Engineering, GITAM School of Technology, Bengaluru campus, Karnataka, India. She received her BE in CSE from Visvesvaraya Technological University in 2013 and her M.Tech in CSE from Visvesvaraya Technological University in 2015. Her area of interest is Cyber security. She has published 3 papers in the international journals.

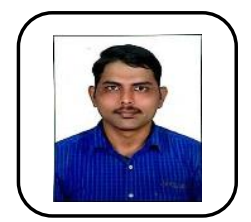

Dr. Dayanand Lal. $\mathbf{N}$ Assistant Professor in CSE Department, GITAM School of Technology, Bengaluru campus. He has published three research papers "Porting presentation Layer to ensure network security in mobile devices", "configuring a secure wireless network using GNS3" and "Protective and Efficacious cloud evaluating Schema” in Scopus journals. The Research interest area is Cyber Security.

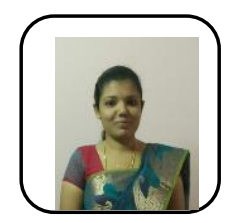

Mrs. Vidya $\mathbf{J}$ is working as an Assistant Professor in the department of Computer Science and Engineering, GITAM School of Technology, Bengaluru campus, Karnataka, India. She received her BE in CSE from Visvesvaraya Technological University in 2013 and her M.Tech in CSE from Visvesvaraya Technological University in 2015. Her area of interest is IoT security and Machine learning. She has published 4 papers in the international journals.

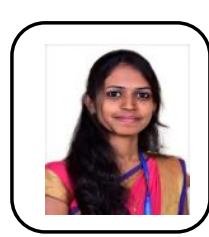

Ms. Bhanujyothi H C is working as an Assistan Professor in the department of Computer Science and Engineering, GITAM School of Technology, Bengaluru campus, Karnataka, India. She received her $\mathrm{BE}$ in CSE from Visvesvaraya Technological University in 2012 and her M.Tech in CSE from Visvesvaraya Technological University in 2014. Her area of interest is IoT security. She has published 3 papers in the international journals. 